\title{
Effect of endotracheal suctioning just after birth in non-vigorous infants born through meconium stained amniotic fluid: A randomized controlled trial
}

\author{
S.N. Singh ${ }^{\mathrm{a}}$, Shifalika Saxena ${ }^{\mathrm{a}}$, Arpita Bhriguvanshi ${ }^{\mathrm{a}, *}$, Mala Kumar $^{\mathrm{a}}$, Chandrakanta $^{\mathrm{a}}$, Sujata ${ }^{\mathrm{b}}$

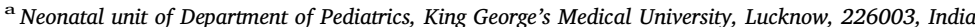 \\ b Department of Obstetrics \& Gynaecology, King George's Medical University, Lucknow, 226003, India
}

\section{A R T I C L E I N F O}

\section{Keywords:}

Endotracheal suctioning

Meconium stained amniotic fluid (MSAF)

Meconium aspiration syndrome (MAS)

Non-vigorous newborn infants

Respiratory distress

\begin{abstract}
A B S T R A C T
Background: Depressed neonates born through meconium stained amniotic fluid (MSAF) are at high risk for developing meconium aspiration syndrome (MAS). Benefits and risk of endotracheal suctioning in non-vigorous infants at birth, though widely practiced till recent have not been established.

Objectives: To evaluate effect of endotracheal suctioning at birth on incidence of MAS, its severity and outcome among non-vigorous MSAF born infants.

Methods: This randomized open level trial was conducted over one year period at a tertiary care teaching hospital. 155 non-vigorous infants (vigour assessed within 5-10 s of birth) were randomized to receive either endotracheal (ET) suctioning just after birth $(n=76)$ or no-endotracheal suction $(n=79)$. Subsequent resuscitation was performed as per neonatal resuscitation guideline. All infants were admitted for subsequent care and monitoring. Antenatal, intrapartum, and neonatal details; respiratory support; complications, hospital stay and outcome were recorded.

Results: Incidence of respiratory distress due to MAS (with consistent chest radiology) was $41.3 \%$ and $57.1 \%$ $[\mathrm{OR}=0.53(0.28$ to 1.01$) ; \mathrm{p}=0.052]$, while non-MAS respiratory distress was $33.3 \%$ and $27.3 \%$ $[\mathrm{OR}=1.69(0.81$ to 3.54$) ; \mathrm{p}=0.17]$ in ET suction and no-ET suction group, respectively. Severity of MAS in ET suction vs. no-ET suction group were mild:16.1\%(5/31)vs15.9\%(7/44); moderate:61.3\%(19/31)vs65.9\%(29/ 44) and severe:22.6\%(7/31)vs18.2\% (8/44). Respiratory support requirement including mechanical ventilation; its duration, and mortality were similar in both groups, however, hospital stay was shorter in ET suction group $(9.91 \pm 3.22 v s .11 .17 \pm 3.73$ days; mean diff: $-1.26(-3.36$ to -0.17$) ; \mathrm{p}=0.024)$.

Conclusions: Endotracheal suctioning at birth in non-vigorous infants born through MSAF tends to decrease the incidence of MAS and duration of hospital stay, however, overall incidence of respiratory distress and mortality remain unchanged.
\end{abstract}

\section{Introduction}

Intrauterine passage of meconium leading to meconium stained amniotic fluid (MSAF) generally signifies underlying fetal hypoxia ${ }^{1}$ and is a risk for meconium aspiration syndrome (MAS). Aspiration of meconium interferes with normal respiratory physiology by causing airway obstruction, chemical irritation, inflammation, surfactant inactivation, meconium induced apoptosis and there is increased risk to develop air leaks, Persistent Pulmonary Hypertension of newborn (PPHN) and sepsis. ${ }^{2},{ }^{3}$ Infants developing MAS exhibit signs of respiratory distress, hypoxemia, carry a high risk of mortality, and long term respiratory and neurodevelepmental morbidities. Incidence of
MSAF ranges from $5.6 \%$ to $24 \%$ (median 14\%) and MAS develops in 1.7 to $35.8 \%$ (median10.8\%). ${ }^{4}$ Depressed neonates born through thick consistency MSAF are at greatest risk for developing MAS. ${ }^{5},{ }^{6}$

There have been several changing recommendations over last few years regarding delivery room management of neonates born through MSAF, based on evidences. ${ }^{7-10}$ Till recent, endotracheal suctioning at birth for non-vigorous infants has been in practice, however, there was a concern of delaying the resuscitation of the already compromised infant, especially in unskilled hands. ${ }^{11}$ Due to lack of evidence of benefits of endotracheal suctioning at birth in non-vigorous infants, recent neonatal resuscitation guidelines do not recommend it as a routine and emphasis was given on initiating ventilation within the first

\footnotetext{
* Corresponding author at: Department of Pediatrics, King George's Medical University, Lucknow, 226003, Uttar Pradesh, India.

E-mail address: arpita@kgmcindia.edu (A. Bhriguvanshi).
} 
minute of life in non-breathing or ineffectively breathing infants. ${ }^{12}$ However, the guidelines also suggest that endotracheal suctioning may be considered if a meconium plug/ airway obstruction is suspected and indicate the need for further study. ${ }^{13}$ We intended to conduct this study to evaluate the effects of immediate postnatal endotracheal suctioning (ET suction) in non-vigorous neonates born through MSAF.

\section{Material and methods}

\subsection{Study design, subjects and setting}

This was an open labeled randomized controlled trial, conducted between September 2011 and August 2012, at the Neonatal and Obstetrics unit of a tertiary care teaching hospital of northern India. A total of 155 singleton newborns infants born through MSAF who were non-vigorous at birth were recruited. Of these, 76 were randomized to 'ET suction group' and 79 to the 'no-ET suction group'. The vigour of the baby was decided within 5-10 s of birth. A neonate was considered vigorous when all of the following met: heart rate $>100 / \mathrm{min}$, reasonably good muscle tone (actively moving or at least flexed extremities) and good respiratory efforts. Newborn, not fulfilling either of these criteria was labeled non-vigorous. ${ }^{11}$ Infants who were vigorous at birth, or $<34$ weeks gestational maturity, or having major congenital malformation and in whom consent could not obtained were excluded. The study was approved by the institutional ethics committee and it was registered at Clinical trial registry of India (CTRI/2011/12/ 002254).

\subsection{Randomization and intervention}

The random allocation sequence was computer generated, and the list bearing the sequence of intervention was kept open, available to the team attending the delivery. Statistician generated the allocation sequence and the pediatric residents enrolled and assigned the participants on fulfilling the eligibility criteria. All the deliveries were attended by at least two pediatric residents who had been trained and skilled in neonatal resuscitation and assisted by staff nurse. Obstetric team used to inform about meconium staining of amniotic fluid in pregnant mothers in labor. Informed written consent was obtained well before delivery from one of the parents to minimize delay between deciding eligibility and commencement of intervention. If MSAF detected on-spot where it was not possible to obtain consent, the neonate was excluded from the trial. If the neonate at birth turned out to be nonvigorous as assessed by the attending pediatric resident team, the infant was recruited and assigned to either ET suction or no-ET suction group in accordance with the random intervention sequence chart. Non-vigorous babies in intervention (ET suction) group were placed under radiant warmer immediately after birth, intubated under direct laryngoscopy and endotracheal suction was performed connecting the other end of infant's endotracheal tube with meconium aspirator which was already kept attached with the suction device (either wall mounted or infant suction device available at newborn corner in delivery room), set at $90-100 \mathrm{~mm} \mathrm{Hg}$ negative pressure. Suction was done while withdrawing the endotracheal tube keeping the suction pressure on, usually for 3-5 s. If meconium was recovered from the trachea and there was no severe bradycardia (heart rate $<60 / \mathrm{min}$ ), the endotracheal suctioning procedure was repeated once again (a maximum of 2 suction attempts). Subsequent to ET suction, oropharyngeal and nasal suction was done using suction catheter, followed by drying, stimulation if required, repositioning and evaluation of breathing and heart rate. Further resuscitation was provided as required, in accordance with NRP guidelines including intubation for positive pressure ventilation (PPV). ${ }^{11}$ Infants allocated to no-ET suction group were immediately placed under warmer, oropharyngeal and then nasal suctioning was performed using 12-14 F suction catheters. Other components of initial steps and any further resuscitation were done as per NRP guidelines including intubation for providing PPV. ${ }^{11}$ Blood gas analysis was done preferably on cord blood sample or arterial blood dawn within 1st hour of life.

\subsection{Data collection and monitoring}

All infants were admitted to the neonatal unit for continuum of care and monitoring, at least for $72 \mathrm{~h}$. Infants monitored for respiratory distress using Downe's score ${ }^{14}$ and for other complications. Management of the infants was according to our unit protocol and equal care was ensured for all. Infants demonstrating respiratory distress were supported on oxygen by head box or continuous positive airway pressure (CPAP), targeting O2 saturation of 92-95\%. Mechanical ventilation was initiated if patient on $\mathrm{O} 2(>80 \%)$ showed arterial PO2 < $50 \mathrm{mmHg}$ or arterial PCO2 $>60$ with $\mathrm{pH}<7.25$ or on clinical decision: Downe's score $>6$ or intractable apneic spells or poor/ no spontaneous respiratory effort. Relevant investigations, including chest radiography, sepsis screen and blood culture were performed in infants developing respiratory distress and in those with risk factor for sepsis, initially at admission and subsequently if required. Echocardiography was done whenever indicated and feasible. Our policy was to wean early from respiratory support, after clinical improvement was evident. Decision to discharge the patient was taken by the attending physician. We discharged the patients once their condition stabilized, completed injectible medication, on total oral feed at least for $48 \mathrm{~h}$ and had normal weight pattern.

Maternal demographic, antenatal, intrapartum, neonatal details including resuscitation and investigative work-up were recorded on a predesigned proforma. The main outcomes measured were development of respiratory distress due to MAS and mortality. Other outcomes were severity of MAS, hypoxic ischemic encephalopathy (HIE) assessed by Levene's stage, ${ }^{15}$ respiratory support required, duration of hospital stay, and any complications: air leak, PPHN. Persons assessing outcome were not aware of intervention group.

\subsection{Study definitions}

MAS: defined as early onset respiratory distress in an neonate born through MSAF whose symptoms could not be otherwise explained and who had characteristic radiological findings (coarse irregular infiltrates, hyperinflation, and or segmental or lobar atelactasis). ${ }^{9}$ MAS severity $^{4}$ : i) mild MAS- requirement of less than $40 \%$ oxygen for $<$ $48 \mathrm{~h}$, ii) moderate MAS-requirement of more than $40 \%$ oxygen or for $>48 \mathrm{~h}$ or on CPAP with no air leak, iii) severe MAS-requirement of assisted ventilation or occurrence of air leak/ PPHN. Prolonged labor: Prolonged labor also known as failure to progress occurs when labor lasts for $20 \mathrm{~h}$ or more in primiparous mother and $14 \mathrm{~h}$ or more in multiparous mother. Obstructed labor: Labour is considered obstructed when the presenting part of the fetus cannot progress into the birth canal, despite strong uterine contractions. Premature rupture of membrane: It was defined as rupture of membranes before the onset of labor. Prolonged rupture of membrane: It was rupture of membranes $>24 \mathrm{~h}$ prior to delivery. Consistency of meconium: It was defined as thin - watery consistency fluid without particles; or thickfluid of pea soup consistency or opaque fluid containing particulate material. Fetal Distress: Evidence of fetal distress was used for nonreassuring fetal heart tracings defined as category III fetal heart rate tracings, which included either 1) absent baseline fetal heart rate variability and any one of the following recurrent late decelerations, recurrent variable decelerations, or bradycardia and 2) sinusoidal pattern. ${ }^{16}$ Respiratory distress: It was diagnosed when one or more of the followings were present: tachypnea ( $R R \geq 60 / \mathrm{min}$ ), chest retraction and expiratory grunting. ${ }^{17}$ PPHN: Diagnosed on the basis of labile hypoxemia (SpO2 reaching below $90 \%$ and/ or arterial PO2 below $50 \mathrm{mmHg}$ ) with pre and post-ductal oxygen saturation difference of $>10 \%$ or arterial PO2 difference of $>20 \mathrm{mmHg}$ with or without echocardiography confirmation. Evidence of PPHN on 
echocardiography was based on demonstration of right-to-left or bidirectional shunting of blood at the foramen ovale and/or the ductus arteriosus as well as high pulmonary arterial/ right ventricular pressure estimated by Doppler velocity measurement of tricuspid regurgitant jet, in absence of other structural cardiac lesions. ${ }^{18}$ Standard definitions were used for other conditions.

\subsection{Sample size and analysis}

We assumed the risk of MAS without intervention to be at a high end of $40 \%$ in high risk non-vigorous infants. A sample size of 76 in each group was calculated to detect a risk difference in MAS of $20 \%$ between the groups, with intervention; which give a power of $80 \%$ and at two sided alpha of $10 \%$. Data analysis was done using SPSS software version 16. Continuous data with normal distribution was analyzed by student $\mathrm{t}$-test and non- normally distributed data by Mann Whitney $\mathrm{U}$ test. Categorical data was analyzed by chi -square or Fisher exact test and binary logistic regression. A p value of 0.05 was taken as significant. Analysis was intention to treat.

\section{Results}

Enrolment of infants in the study is depicted in Fig.1. Of 155 nonvigorous newborn infants, 76 were randomized to ET suction group and 79 to no-ET suction group. One infant in each group detected to have congenital heart disease and one in no- ET suction group had congenital diaphragmatic hernia, thus excluded from analysis. Maternal demographic, antenatal, intrapartum parameters including mode of delivery and consistency of meconium were similar in both groups ( $p>0.05$ ) (Table1). Post-term deliveries were one in each group. Mean birth weight, gestational age and gender distribution of neonates were similar in both groups (Table 2). Repeat endotracheal suctioning was done in $21(28 \%)$ infants. Twenty (26.7\%) infants in ET suction group
Table 1

Maternal and Intrapartum parameters.

\begin{tabular}{llll}
\hline Parameters & $\begin{array}{l}\text { ET suction } \\
\text { group }(\mathrm{N}=75)\end{array}$ & $\begin{array}{l}\text { No ET suction } \\
\text { group (N = 77) }\end{array}$ & p-value \\
\hline Maternal age (yr), mean \pm sd & $\begin{array}{l}27.1 \pm 3.6 \\
33(44 \%)\end{array}$ & $26.6 \pm 3.7$ & 0.33 \\
Multigravida & $49(65.3 \%)$ & $57(74 \%)$ & 0.51 \\
Adequate ANC & $6(8 \%)$ & $5(6.5 \%)$ & 0.24 \\
Post-dated pregnancy(> 40 w) & $23(30.7 \%)$ & $19(24.7 \%)$ & 0.76 \\
PIH/preeclampsia/eclampsia & $12(16 \%)$ & $9(11.7 \%)$ & 0.41 \\
Anemia (Hb < 10 g/dl) & $19(25.3 \%)$ & $23(29.9 \%)$ & 0.44 \\
Premature rupture of membrane & $13(16.8 \%)$ & 0.94 \\
Prolonged rupture of membrane & $13(17.3 \%)$ & $21(27.3 \%)$ & 0.17 \\
Prolonged / Obstructed labor & $29(38.7 \%)$ & $9(11.7 \%)$ & 1.0 \\
Other complications & $8(10.7 \%)$ & $33(42.9 \%)$ & 0.76 \\
Fetal distress & $34(45.3 \%)$ & & \\
Mode of delivery & & $18(23.4 \%)$ & 0.17 \\
$\quad$ Vaginal spontaneous & $26(34.7 \%)$ & $29(37.7 \%)$ & \\
$\quad$ Vaginal assisted & $19(25.3 \%)$ & $30(39 \%)$ & 0.87 \\
$\quad$ Caesarean & $30(40.0 \%)$ & $47(39.0 \%)$ & \\
Thick meconium & $47(37.3 \%)$ & & \\
\hline
\end{tabular}

All the data is represented as n(\%) unless specified; Adequate antenatal care (ANC): Registered pregnancy with four or more antenatal visits was considered as adequate ANC; PIH:Pregnancy induced hypertension; Other complications: Gestational diabetes on insulin(1:1),Oligohydramnios (2:1) Hypothyroidism (1:1), Rheumatic heart disease( $0: 1)$, Urinary tract infection (4:5) in ET-suction \& No-ET suction group respectively.

and $23(29.9 \%)$ in no-ET suction group were intubated in delivery room for PPV. Respiratory distress developed in 56 infants among ET suction group [MAS: 31, unexplained (non-MAS):25] and 65 infants in no-ET suction group [MAS: 44, unexplained (non-MAS):21]. The incidence of MAS was lesser in ET suction group [31(41.3\%) vs. 44(57.1\%); $\mathrm{OR}=0.53(0.28$ to 1.01$) ; \mathrm{p}=0.052]$. There was no significant difference in number of cases with mild $(5 / 31,16.1 \%$ vs. $7 / 44,15.9 \%)$,

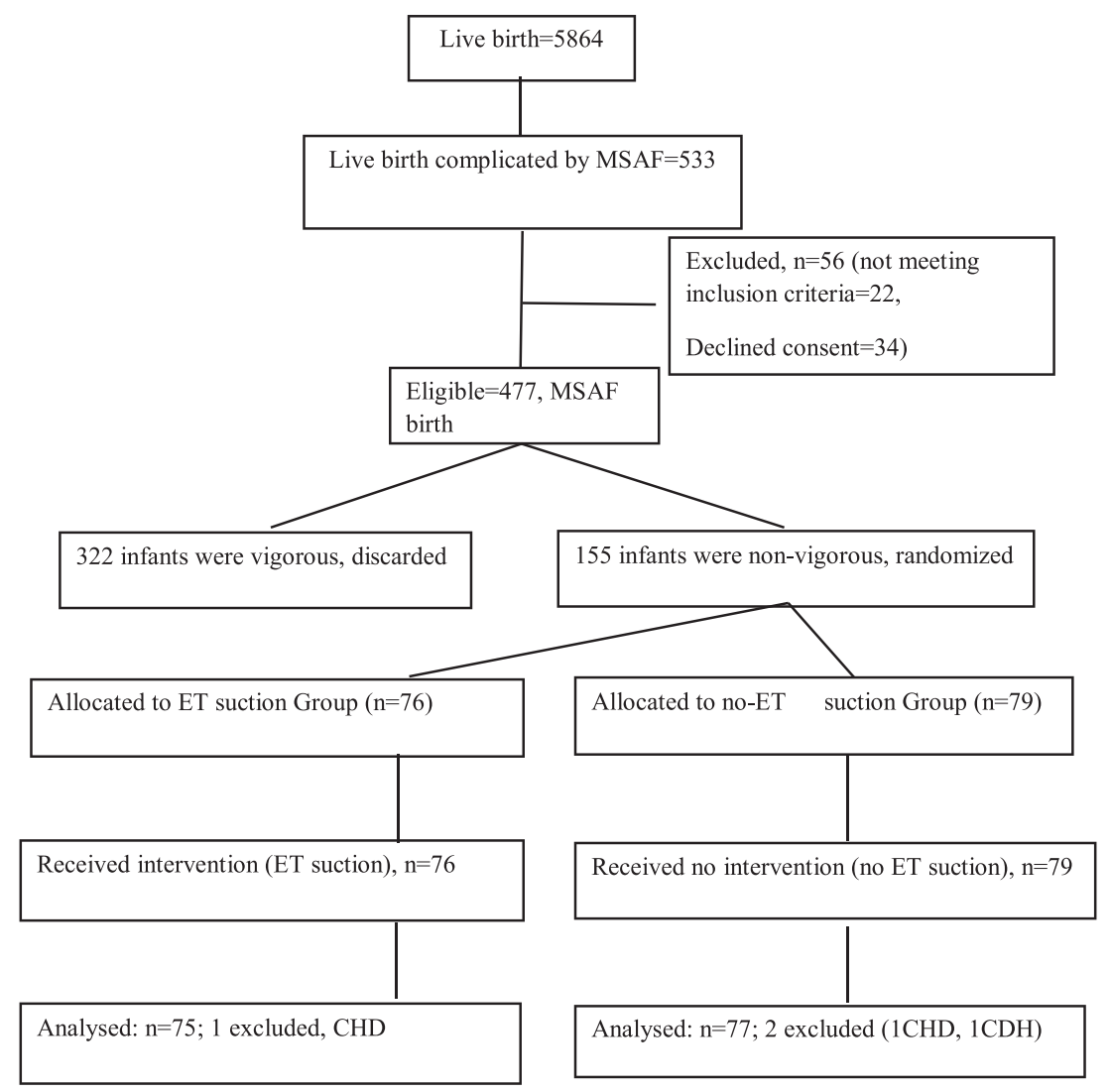

Fig. 1. CONSORT diagram. Flow of the participants in the study. 
Table 2

Neonatal parameters.

\begin{tabular}{|c|c|c|c|}
\hline Parameters & ET suction group $(\mathrm{N}=75)$ & No ET suction group $(\mathrm{N}=77$ ) & p-value \\
\hline Birth weight ( $g$; mean \pm sd) & $2462 \pm 315$ & $2461 \pm 192$ & 0.97 \\
\hline Low birth weight & $28(37.3 \%)$ & $32(41.6 \%)$ & \\
\hline Gestational maturity (wk; mean \pm sd) & $38.57 \pm 2.2$ & $38.56 \pm 2.0$ & 0.96 \\
\hline Male: Female & $40: 35$ & $43: 34$ & 0.75 \\
\hline \multicolumn{4}{|l|}{ Resuscitation } \\
\hline Initial steps only & $17(22.7 \%)$ & $16(20.8 \%)$ & 0.98 \\
\hline $\mathrm{PPV} \leq 1 \mathrm{~min}$ & $22(29.3 \%)$ & $21(27.3 \%)$ & \\
\hline $\mathrm{PPV}>1 \mathrm{~min}$ & $31(41.3 \%)$ & $35(45.5 \%)$ & \\
\hline Chest compression & $4(5.3 \%)$ & $4(5.2 \%)$ & \\
\hline Epinephrine Inj & $1(1.3 \%)$ & $1(1.3 \%)$ & \\
\hline APGAR Score at $1 \mathrm{~min}$ (median,IQR) & $4(3-5)$ & $4(3-5)$ & 0.37 \\
\hline APGAR Score at $1 \mathrm{~min}: \leq 3$ & $29(38.7 \%)$ & $25(32.5 \%)$ & \\
\hline $4-6$ & $41(54.6 \%)$ & $46(59.75)$ & \\
\hline$\geq 7$ & $5(6.7 \%)$ & $6(7.8 \%)$ & \\
\hline APGAR Score at 5 min (median,IQR) & $7(6-7)$ & $7(6-7)$ & 0.29 \\
\hline APGAR Score at $5 \mathrm{~min}: \leq 3$ & $1(1.3 \%)$ & $0(0)$ & \\
\hline $4-6$ & $29(38.7 \%)$ & $26(33.8 \%)$ & \\
\hline$\geq 7$ & $45(60 \%)$ & $51(66.2 \%)$ & \\
\hline Cord $/<1 \mathrm{~h}$ arterial blood $\mathrm{pH}$ (mean \pm sd) & $7.084 \pm 0.111$ & $7.087 \pm 0.110$ & 0.84 \\
\hline Cord blood $\mathrm{pH}:<7.0$ & $10(13.7 \%)$ & $16(21.1 \%)$ & \\
\hline $7-7.2$ & $53(72.6 \%) 10$ & $52(68.4 \%)$ & \\
\hline$>7.2$ & $(13.7 \%)$ & $7(10.3 \%)$ & \\
\hline missing & 2 & 1 & \\
\hline Base deficit (mean \pm sd) & $15.86 \pm 3.22$ & $15.77 \pm 3.13$ & 0.85 \\
\hline Base deficit: $>16$ & $43(58.9 \%)$ & $33(43.4 \%)$ & \\
\hline $12-16$ & $22(30.1 \%)$ & $32(42.1 \%)$ & \\
\hline$<12$ & $8(11 \%)$ & $11(14.5 \%)$ & \\
\hline
\end{tabular}

All the data is represented as $\mathrm{n}(\%)$ unless specified.

Table 3

Outcome parameters.

\begin{tabular}{|c|c|c|c|c|}
\hline Parameters & ET Suction group $(\mathrm{N}=75)$ & No ET Suction group $(\mathrm{N}=77)$ & Odds Ratio/mean diff (95\% CI) & $\mathrm{p}$-value \\
\hline MAS & $31(41.3 \%)$ & $44(57.1 \%)$ & $0.53(0.28-1.01)$ & 0.05 \\
\hline \multicolumn{5}{|l|}{ MAS severity } \\
\hline Mild & $5(6.7 \%)$ & $7(9.3 \%$ & & \\
\hline Moderate & $19(25.3 \%)$ & $29(38.7 \%)$ & & 0.94 \\
\hline Severe & $7(9.3 \%)$ & $8(10.7 \%)$ & & \\
\hline HIE: Moderate/ Severe & $20(26.7 \%)$ & $30(39.0 \%)$ & $0.57(0.29-1.13)$ & 0.11 \\
\hline \multicolumn{5}{|l|}{ Sepsis } \\
\hline Culture positive & $3(4 \%)$ & $1(1.3 \%)$ & $3.12(0.32-30.7)$ & 0.33 \\
\hline Screen positive & $5(6.7 \%)$ & $6(7.8 \%)$ & $0.93(0.27-3.19)$ & 0.90 \\
\hline Pneumothorax & $3(4 \%)$ & $2(2.6 \%)$ & $0.51(0.04-5.71)$ & 0.58 \\
\hline PPHN & $6(8.0 \%)$ & $7(9.1 \%)$ & $0.87(0.28-2.72)$ & 0.81 \\
\hline Secondary pneumonia & $17(22.7 \%)$ & $19(24.7 \%)$ & $0.97(0.45-2.07)$ & 0.93 \\
\hline Resp support needed(included MV) & $65(86.7 \%)$ & $69(89.6 \%)$ & $0.75(0.28-2.03)$ & 0.57 \\
\hline Duration of Resp support (days; mean \pm sd) & $2.36 \pm 1.52$ & $2.65 \pm 1.43$ & $-0.29(-0.79$ to 00.21$)$ & 0.26 \\
\hline Need for MV & $11(14.7 \%)$ & $13(16.9 \%)$ & $0.85(0.35-2.03)$ & 0.71 \\
\hline${ }^{\mathrm{a}}$ Duration of MV (day; mean \pm sd) & $3.27 \pm 1.10$ & $3.46 \pm 0.66$ & $-0.19(-0.94$ to 0.57$)$ & 0.61 \\
\hline${ }^{\mathrm{a}}$ Duration of hospital stay (days; mean \pm sd) & $9.91 \pm 3.06$ & $11.17 \pm 3.73$ & $-1.26(-2.36$ to -0.17$)$ & 0.02 \\
\hline Mortality/LAMA & $4(5.3 \%)$ & $7(9.1 \%)$ & $0.56(0.16-2.01)$ & 0.38 \\
\hline
\end{tabular}

All the data is represented as n(\%) unless specified; MV:Mechanical ventilation.

${ }^{a}$ Duration of $>12 \mathrm{~h}$ was recorded as an day.

moderate $(19 / 31,61.3 \%$ vs. $29 / 44,65.9 \%)$ or severe MAS $(7 / 31$, $22.6 \%$ vs. $8 / 44,18.2 \%$ ) among ET suction vs. no-ET suction group. Of infants developing respiratory distress, nine (16.0\%) in ET suction group and four (6.0\%) in no-ET suction group required O2 for less than $12 \mathrm{~h}$. Number of cases requiring respiratory support and the mean duration of respiratory support were similar in both groups (Table3). Similarly, requirement of mechanical ventilation and its mean duration were comparable between both groups. However, the mean duration (days; mean \pm sd) of hospital stay was shorter in ET suction group $[9.91 \pm 3.22$ vs. $11.17 \pm 3.73$; mean diff: $-1.26(-3.36$ to -0.17$)$; $\mathrm{p}=0.024]$. In infants born through thick consistency of meconium, the risk of MAS was comparable in both groups (ET suction: 21/47 (44.7\%), no-ET suction: $30 / 47(63.8 \%) ; \mathrm{p}=0.09)$. None of the patient had intubation related complications. None of the infants in either group received surfactant.

Incidence of non-MAS (unexplained) respiratory distress was $33.3 \%$ and $27.3 \%$ [OR $=1.69(0.81-3.54) ; \mathrm{p}=0.17]$ in ET suction and no-ET suction group, respectively. On analysis of non-MAS respiratory distress cases, the baseline parameters were comparable between the two groups. The mean duration of respiratory support (days) $[2.11 \pm 1.43$ vs. $2.45 \pm 1.23$; mean diff $-0.34(-1.17$ to 0.50 ; $p=0.42)$ ], need of mechanical ventilation ( $3 / 22$ vs. $2 / 20$;), mean duration of mechanical ventilation (days) $[3.67 \pm 1.52$ vs. $4.0 \pm 0.01$; mean diff $-0.33(-3.95$ to 3.29$) ; \mathrm{p}=0.79$, mean duration of hospital stay (days) $[9.68 \pm 3.31$ vs. $11.35 \pm 3.36$; mean diff $-1.67(-3.75$ to $0.41) ; \mathrm{p}=0.11$, and mortality/ LAMA ( $3 / 22$ vs. $2 / 20 ; \mathrm{p}=1.0$ ) were 
comparable between ET suction and no-ET suction group, respectively. Similarly, the baseline parameters of the infants developing MAS were comparable between the two groups. The outcome parameters including mean duration of respiratory support (days) $[2.68 \pm 1.56 \mathrm{vs}$. $2.84 \pm 1.54$; mean diff $-0.16(-0.89-0.56 ; \mathrm{p}=0.65)$ ], need of mechanical ventilation ( $3 / 31$ vs. $11 / 44 ;)$, mean duration of mechanical ventilation (days) $[3.0 \pm 1.0$ vs. $3.36 \pm 0.67 ;$ mean diff $-0.36(-1.20-0.47) ; \mathrm{p}=0.37]$, mean duration of hospital stay (days) [10.42 \pm 2.85 vs. $11.45 \pm 3.81$; mean diff $-1.03(-2.65-0.58)$; $\mathrm{p}=0.20$, and mortality/ LAMA ( $1 / 31$ vs. $5 / 44 ; \mathrm{p}=0.39)$ were similar in ET suction and no-ET suction group, respectively.

\section{Discussion}

Intrauterine asphyxia, infection and postmaturity often lead to passage of meconium by the fetus. Normally, fetal breathing activity results in movement of lung fluid out of the trachea. ${ }^{19}$ However, prolonged/ severe fetal stress may stimulate fetal breathing and gasping, can lead to aspiration of MSAF or aspiration can occur during initial breaths after delivery. ${ }^{20}$ The extent to which meconium has reached the distal airways by the time of birth and the effect of tracheal suctioning of meconium just after birth in preventing or minimizing the respiratory complications has still not been established. Our finding showed a marginal reduction in incidence of MAS among non-vigorous infant undergoing ET suction $[41.3 \%$ vs. 57.1\%; $\mathrm{OR}=0.53(0.28-1.01) ; \mathrm{p}=0.052]$ and thus it supports the possibility of aspiration during initial breaths after birth which can be minimized by prompt postnatal tracheal suctioning. Our finding differ from the other two Indian reports, conducted on non-vigorous infants born through MSAF. ${ }^{21},{ }^{22}$ Chettri et al. ${ }^{21}$ randomized 122 non-vigorous infants born through MSAF to tracheal suction and no-suction groups and the incidence of MAS reported was similar in both groups (33\% vs. $31 \% ; \mathrm{p}=1.0$ ). In a trial of 175 non-vigorous infants born through MSAF, Nangia et al. ${ }^{22}$ reported the incidence of MAS to be $32.3 \%$ in ET suction group and $26.1 \%$ in no-ET suction group ( $p=0.38$ ). The difference in incidence of MAS among these studies and ours may be due to variation in methodology, patient profile and perinatal care. In Nangia et al. ${ }^{22}$ study, the infants in ET suction group got intervention only after oral \& nasopharyngeal suctioning which may have defeated the purpose as it might have stimulated and initiated breathing efforts before the task of tracheal suctioning

We find a high rate of non-MAS (unexplained) respiratory distress cases, not demonstrating typical radiologic finding of MAS [ET-suction group: $33.3 \% \& \quad$ no-ET-suction: $27.3 \%$; OR $=1.69(0.81-3.54)$; $\mathrm{p}=0.17]$. Chettri et $\mathrm{al}^{21}$ reported respiratory distress ranging from mild to severe in $95.9 \%$ of their infants at the time of NICU admission, however, MAS only in $32 \%$. Fleischer at $\mathrm{al}^{23}$ and Yoder et $\mathrm{al}^{24}$ had described non-MAS respiratory distress to be a common among infants born through MSAF compared to those born through clear meconium. These non-MAS respiratory disorders may actually be the part of overall spectrum of MAS and there may not be association between the degree of radiologic abnormalities and the severity of MAS. ${ }^{9}$

One of the important concerns with practice of ET suctioning is delaying resuscitation of the already compromised infants which may potentially enhance asphyxial injuries. However, we did not find any increase in the rate of PPV, chest compression or adrenaline requirement at birth, support on mechanical ventilation, complications and mortality in infants undergoing ET suctioning; alleviating the concern. These observations were similar to that of Chettri et al., ${ }^{21}$ who also reported mental developmental delay at 9 months, to be similar in the ET-suction $(24 \%, 10 / 42)$ and no-ET suction group (32\%, 14/44). However, Nangia et al. ${ }^{22}$ reported higher rate of bag and mask ventilation in infants of no-ET suction group compared to ET suction group $(73.9 \%$ vs. $59.8 \% ; \mathrm{p}=0.48)$ and they related it to the timing of evaluation and possible stimulation during the ET suctioning. PPHN was detected in 13 infants; however, it could not be confirmed by echocardiography in majority of our patients due to logistic reasons.

The duration of hospital stay of patients with MAS have been found to be variable in different studies depending on patient profile, complications, level of care and discharge policy. ${ }^{2},{ }^{25}$ In our study, patients undergoing endotracheal suctioning had shorter mean hospital stay compared to those without endotracheal suction $(9.91 \pm 3.22$ vs. $11.17 \pm 3.73$ days; mean diff: $-1.26(-3.36$ to -0.17$) ; \mathrm{p}=0.024)$. This trend was similar that reported by Chettri et al. ${ }^{21}(2.7 \pm 2.2$ days in suction group vs. $3.4 \pm 3.1$ in no-suction group; $p=0.18$ ). Since other risk factors and complications were distributed equally in both ET suction and no-ET suction group in our study, we postulated that removing the residual meconium from trachea by endotracheal suctioning at birth may have reduced the burden of meconium aspiration and its consequences resulting in shorter stay in hospital. Though, meconium was recovered from endotracheal suctioning in nearly all of our patients, it was not quantified. Nangia et al. ${ }^{22}$ did not find difference in mean duration of hospital stay in patients' undergoing ET suction and no-ET suction $(2.99 \pm 1.26$ vs. $2.95 \pm 0.88$ days; $\mathrm{p}=0.42$ ). The variation in duration of hospital stay among studies by Chhetri et al. ${ }^{21}$, Nangia et al. ${ }^{22}$ and ours may be because of differences in patient profile, associated co-morbidities/ complications and discharge policy.

The strength of our study include the randomized controlled design, and adherence to protocol, however, it had many limitations. The study was not blinded because of nature of the intervention and limited number of staff; however, it was attempted at level of outcome measurement. The sample size taken was small because of the limited time frame available for the conduct of study and it was calculated to detect a modest difference in incidence of MAS in the two groups. Because of small sample size, subgroup analysis was not possible, which otherwise seems meaningful.

\section{Conclusion}

Our study suggest that endotracheal suctioning immediately after birth in non-vigorous infants born through meconium stained amniotic fluid tends to decreases the incidence of MAS and duration of stay in hospital, though, overall incidence of respiratory distress, severity of MAS, and mortality remain unchanged. These findings need confirmation in other settings and more specified population of non-vigorous newborn infants.

\section{References}

1. Monen L, Hasaart TH, Kuppens SM. The aetiology of meconium-stained amniotic fluid: pathologic hypoxia or physiologic foetal ripening? Review. Early Hum Dev. 2014;90(7):325-328.

2. Louis D, Sundaram V, Mukhopadyay K, Dutta S, Kumar P. Predictors of mortality in neonates with meconium aspiration symdrome. Indian Pediatr. 2014;51(8):637-640.

3. Fischer C, Rybakowski C, Ferdynus C, Sagot P, Gouyon JB. A population base study of meconium aspiration syndrome in neonates born between 37 and 43 weeks of gestation. Int J Pediatr. 2012;72(5):425-428.

4. Cleary GM, Wiswell TE. Meconium -stained amniotic fluid and the meconium aspiration syndrome: an update. Pediatr Clin North Am. 1998;45:511-529.

5. Wiswell TE. Delivery room management of the meconium stained newborn. $J$ Perinatol. 2008;28:S19-S26.

6. Bhat RY, Rao A. Meconium stained amniotic fluid and meconium aspiration syndrome: a prospective study. Ann Trop Pediatr. 2008;28:199-203.

7. Vain NE, Szyld EG, Prudent LM, et al. Oropharyngeal and nasopharyngeal suctioning of meconium-stained neonates before delivery of their shoulders: multicentre, randomised controlled trial. Lancet. 2004;364:597.

8. Roggensack A, Jefferies AL, Farine D, et al. Management of meconium at birth. $J$ ObstetGynaecol Can. 2009;31:353.

9. Wiswell TE, Gannon CM, Jacob J, et al. Delivery room management of the apparently vigorous meconium stained neonate: results of the multicentre international collaborative trial. Pediatrics. 2000;105:1-7.

10. Halliday HL, Sweet DG. Endotracheal intubation at birth for preventing morbidity and mortality in vigorous, meconium-stained infants born at term. Cochrane Database Syst Rev. 2001(1) CD000500.

11. Kattwinkel J, Perlman JM, Aziz K, et al. Part 15: neonatal resuscitation: 2010 American Heart Association Guidelines for cardiopulmonary resuscitation and emergency cardiovascular care. Circulation. 2010;122:S909-S919. 
12. Wyckoff MH, Aziz K, Escobedo MB, et al. Part 13: neonatal resuscitation: 2015 American Heart Association Guidelines update for cardiopulmonary resuscitation and emergency cardiovascular care. Circulation. 2015;132:S543-S560.

13. Perlman JM, Wyllie J, Kattwinkel J, et al. Part 7: neonatal resuscitation: 2015 international consensus on cardiopulmonary resuscitation and emergency cardiovascular care science with treatment recommendations. Circulation. 2015;132:S204-S241.

14. Downes JJ, Vidyasagar D, Boggs TR, Morrow GM. Respiratory distress syndrome of newborn infants. I. New clinical scoring system (RDS score) with acid base and blood gas correlations. Clin Pediatr. 1970;9:325-331.

15. Levene ML, Kornberg J, Williams TH. The incidence and severity of post-asphyxial encephalopathy in full-term infants. Early Hum Dev. 1985;11(1):21-26.

16. ACOG practice bulletin No. 106: intrapartum fetal heart rate monitoring nomenclature, interpretation, and general management principles. Obstet Gynecol. 2009;114(1):192-202.

17. NNPD network. National neonatal perinatal database- report for the year 2002-2003 New Delhi: NNF NNPD network; 2005 Ref type: Report.

18. Rychik J, Cohn MS. Echocardiography in the neonatal intensive care unit. In: Taeusch HW, Ballard RA, Gleason CA, eds. Avery's diseases of the newborn. 8th ed.
Philadelpha: Elsevier; 2005:802-811.

19. Dawes GS, Fox HE, Leduc BM, et al. Respiratory movements and rapid eye movement sleep in the foetal lamb. J Physiol. 1972;220:119-143.

20. Brown BL, Gleicher N. Intrauterine meconium aspiration. Obstet Gynecol. 1981;57:26-29.

21. Chettri S, Adhisivam B, Bhat BV. Endotracheal suction for nonvigorous neonates born through meconium stained amniotic fluid: a randomized controlled trial. J Pediatr. 2015;166:1208.

22. Nangia S, Sunder S, Biswas R, Saili A. Endotracheal suction in term non vigorous meconium stained neonates-a pilot study. Resuscitation. 2016;105:79.

23. Fleischer A, Anyaegbunam A, Guidetti D, et al. A persistent clinical problem: profile of the term infant with significant respiratory complications. Obstet Gynecol. 1992;79:185-190.

24. Yoder BA. Meconium-stained amniotic fluid and respiratory complications: impact of selective tracheal suction. Obstet Gynecol. 1994;83:77-84.

25. Shaikh M, Ahmad K, Waheed KAI, et al. Detrimental complications of meconium aspiration syndrome and their impact on outcome. J Ayub Med Coll Abbottabad. 2016;28:506-509. 